\title{
First identification of Francisella noatunensis subsp. orientalis causing mortality in Mexican tilapia Oreochromis spp.
}

\author{
Cesar Ortega ${ }^{1, *}$, Gerardo Mancera $^{1}$, Ricardo Enríquez ${ }^{2}$, Augusto $\operatorname{Vargas}^{2,3}$, \\ Simón Martínez ${ }^{1}$, Raúl Fajardo', Ruben Avendaño-Herrera ${ }^{3,4,5}$, \\ María José Navarrete ${ }^{2}$, Alex Romero ${ }^{2,3}$ \\ ${ }^{1}$ Centro de Investigación y Estudios Avanzados en Salud Animal (CIESA), Facultad de Medicina Veterinaria y Zootecnia, \\ Universidad Autónoma del Estado de México, Carretera Toluca-Atlacomulco Km. 15.5, Toluca 50200, Mexico \\ ${ }^{2}$ Laboratorio de Biotecnología y Patología Acuática, Instituto de Patología Animal, Facultad de Ciencias Veterinarias, \\ Campus Isla Teja, Universidad Austral de Chile, Independencia 641, Valdivia 5090000, Chile \\ ${ }^{3}$ Centro FONDAP, Interdisciplinary Center for Aquaculture Research (INCAR), PO Box 160-C, Concepción 4030000, Chile \\ ${ }^{4}$ Laboratorio de Patología de Organismos Acuáticos y Biotecnología Acuícola, Facultad de Ciencias Biológicas, \\ Universidad Andrés Bello, 2520000 Viña del Mar, Chile \\ ${ }^{5}$ Centro de Investigación Marina Quintay (CIMARQ), Universidad Andres Bello, Quintay, Chile
}

\begin{abstract}
Francisellosis, an emerging disease in tilapia Oreochromis spp., is caused by the facultative, intracellular bacterium Francisella noatunensis subsp. orientalis, which is present in various countries where tilapia farming is commercially important. We confirmed the presence of francisellosis in Mexican tilapia cultures in association with an outbreak during the second semester of 2012. Broodstock fish presented a mortality rate of approximately $40 \%$, and disease was characterized by histologically classified granulomas, or whitish nodules, in different organs, mainly the spleen and kidney. Through DNA obtained from infected tissue and pure cultures in a cysteine heart medium supplemented with hemoglobin, F. noatunensis subsp. orientalis was initially confirmed through the amplification and analysis of the 16S rRNA gene and the internal transcribed spacer region. Phylogenetic analysis of these genes demonstrated close similarity with previously reported $F$. noatunensis subsp. orientalis sequences obtained from infected tilapia from various countries. The identification of this subspecies as the causative agent of the outbreak was confirmed using the iglC gene as a target sequence, which showed $99.5 \%$ identity to 2 F. noatunensis subsp. orientalis strains (Ethime-1 and Toba04). These findings represent the first documented occurrence of francisellosis in Mexican tilapia cultures, which highlights the importance of establishing preventative measures to minimize the spread of this disease within the Mexican aquaculture industry.
\end{abstract}

KEY WORDS: Francisellosis $\cdot$ Freshwater aquaculture $\cdot$ Mexico $\cdot$ Tilapia

\section{INTRODUCTION}

In Mexico, tilapia Oreochromis spp. comprise the most important freshwater aquaculture species, and in terms of the national fishing industry, tilapia production ranks fourth, surpassed only by sardines, shrimps, and tuna (Conapesca 2012). Currently, almost all of the tilapia produced in Mexico is sold within the country; however, proximity to the North American market and the availability of water resources mean that Mexico has the opportunity to become an international leader in tilapia production 
and commerce (Fitzsimmons 2000). To achieve this, the Mexican aquaculture industry requires tools and processes that favor development and expansion, including the timely diagnosis of infectious diseases (Ortega \& Valladares 2015).

Tilapia species are ideal for aquaculture due to rapid growth rates, resistance to stress caused by handling, tolerance to suboptimal water qualities, and low susceptibility to diseases (Fitzsimmons 2000). Nevertheless, as with other species subjected to culturing systems, tilapia can be affected by diseases, with vulnerability increasing depending on production intensity (Ottem et al. 2009). In relation to this, francisellosis is an emerging disease among tilapia and other fish species that affects wild and cultivated individuals alike in various regions of the world (Nylund et al. 2006, Olsen et al. 2006, Ostland et al. 2006, Mikalsen et al. 2007, Ottem et al. 2009, Soto et al. 2011).

In tilapia, francisellosis is caused by the Gram-negative, facultative intracellular, immobile, strictly aerobic, and highly infectious coccobacillus bacterium Francisella noatunensis subsp. orientalis. This bacterium can affect fish during different developmental stages in fresh and brackish waters (Soto et al. 2009b). Fish affected by francisellosis initially demonstrate decreased activity, slow swimming, and a loss of appetite. Normally, no external abnormalities are evident, although in some cases, fish can present external non-specific abnormalities/lesions (Hsieh et al. 2006, Colquhoun \& Duodu 2011). As the infection advances, live fish can have increased, decreased, or abnormal operculum movement on the surface of the water column or near aeration ducts. Moreover, skin lesions and frayed fins may occur (Mauel et al. 2007). The necropsy of infected fish often reveals splenomegaly with the presence of distinctly sized whitish nodules, a situation that can also occur in the kidney, liver, and, to a lesser extent, in the heart, gonads, intestine, and gills. Histologically, this disease is characterized by the presence of granulomatous lesions in the infected organs (Mauel et al. 2005, Olsen et al. 2006, Soto et al. 2009b).

Francisellosis has not been previously registered in Mexico. However, here we describe the clinical symptoms and macro- and microscopic lesions found in association with a francisellosis outbreak in a tilapia farm in central Mexico during the second semester of 2012. The causative bacterium of this outbreak was identified through culture isolation and confirmed by polymerase chain reaction (PCR) analysis and sequencing of the 16S rRNA gene and internal transcribed spacer region (ITS).
This finding underscores the urgent need for updated management and disease detection practices in the Mexican aquaculture industry to prevent future outbreaks and the spread of francisellosis within tilapia farms.

\section{MATERIALS AND METHODS}

\section{Tissue samples}

In November 2012, a granulomatous disease was reported in tilapia weighing between 200 and $350 \mathrm{~g}$ at a fish farm in central Mexico. Farm personnel detected this disease, observing lower feed intake, less activity, surface swimming, abdominal distention, skin darkening, and mortality in approximately $40 \%$ of the tilapia population. During sample collection, which occurred approximately 1 mo after the outbreak, no mortalities were observed, but the affected fish did present irregular sizes and severe emaciation. Individuals were maintained in $10 \times 4 \mathrm{~m}$ tanks with a daily $10 \%$ change in the water supply and average temperature of $26^{\circ} \mathrm{C}$. Thirty fish with a delayed escape response and/or poor body condition were collected and transported to the Aquatic Animal Health Laboratory of the Facultad de Medicina Veterinaria y Zootecnia in Toluca, Mexico, for a complete pathological and bacteriological workup.

All fish were euthanized through anesthesia overdose and immediately subjected to postmortem examination. Initially, each diseased fish was examined for the presence of external parasites, and scrapings were obtained from the gills and skin for microscopic observation with $10 \times$ and $40 \times$ objectives. Internally, the aspect, color, and content of the body cavity were reviewed. For microscopic analysis, spleen and kidney imprints of the affected specimens were also taken, and the microscope slides were Gram stained.

\section{Histological analysis}

For histological analysis, samples of liver, spleen, kidney, heart, gills, brain, and intestine were taken from each fish, fixed in vials containing $10 \%$ buffered formalin, dehydrated, and embedded in paraffin wax following standard procedures. Each tissue was sectioned at $5 \mu \mathrm{m}$ and stained with hematoxylin and eosin to describe histopathological alterations (Fernando et al. 1972). Sections were observed at different magnifications under an Olympus $\mathrm{BH} 2$ light microscope. 


\section{Bacteriological analysis}

Samples for bacterial isolation were aseptically taken from the spleen, liver, and kidney of each infected fish and streaked onto Columbia blood agar, tryptone soya agar, MacConkey agar, brain heart infusion agar, and cysteine heart agar supplemented with $2 \%$ bovine hemoglobin (CHAB) plates. All plates were aerobically incubated at $26^{\circ} \mathrm{C}$ for 2 to $5 \mathrm{~d}$ (Soto et al. 2011). A representative isolate colony was then selected from each CHAB plate, streaked onto a new $\mathrm{CHAB}$ plate to obtain pure cultures, and stored at $-80^{\circ} \mathrm{C}$ in Criobille tubes (AES Laboratoire).

PCR analysis was used to determine if the presumed etiologic agent corresponded to the Francisella genus. For this, spleen and kidney samples were also taken directly from 10 fish with granulomatous lesions and deposited in $10 \mathrm{ml}$ tubes containing $5 \mathrm{ml}$ of absolute ethanol until further DNA extraction.

\section{DNA extraction}

Total DNA was extracted from spleen and kidney samples, as well as from pure bacterial isolates recovered from colonies cultured on $\mathrm{CHAB}$ plates using the E.Z.N.A. ${ }^{\circledR}$ Tissue DNA Kit (Omega Bio-Tek) according to the manufacturer's instructions. The obtained DNA was stored at $-20^{\circ} \mathrm{C}$ until PCR analysis.

\section{Amplification of the 16S rRNA gene and ITS region of Francisella spp.}

To confirm the Francisella genus, total DNA extracted from tissue samples and each isolate were tested through PCR amplification using the primers
F1 and F5 (Forsman et al. 1994), which resulted in an amplification product of 1100 base pairs (bp). Amplification was carried out in a total reaction volume of $25 \mu \mathrm{l}$, composed of $5 \times$ GoTaq ${ }^{\circledR}$ Flexi Buffer (Promega), $25 \mu \mathrm{M}$ of $\mathrm{MgCl}_{2}, 12.5 \mu \mathrm{M}$ of the each primer set (Table 1), $10 \mu \mathrm{M}$ of dNTPs mix, $2.5 \mathrm{U}$ of $\mathrm{GoTaq}^{\circledR}$ Flexi DNA (Promega), and 250 ng of each DNA template. Amplification was performed with the MultiGene $^{\mathrm{TM}}$ Gradient Thermal Cycler (Labnet) using the following settings: initial denaturalization at $94^{\circ} \mathrm{C}$ for $3 \mathrm{~min}$; followed by 35 cycles at $94^{\circ} \mathrm{C}$ for $30 \mathrm{~s}, 60^{\circ} \mathrm{C}$ for $1 \mathrm{~min}$, and $72^{\circ} \mathrm{C}$ for $1 \mathrm{~min}$; with a final extension at $72^{\circ} \mathrm{C}$ for 5 min. Genomic DNA from an $F$. noatunensis subsp. orientalis strain was included as a positive control for each PCR assay. Negative controls consisted of the same reaction mixture but with sterile distilled water instead of template DNA.

The complete 16S rRNA gene was also obtained from each pure bacterial isolate using the universal primer pair UN and EB (Table 1), which generate a PCR product of approximately 1500 bp (Barry et al. 1990). The PCR reaction was performed using GoTaq ${ }^{\circledR}$ DNA Polymerase (Promega) in a final volume of $25 \mu \mathrm{l}$, which contained $50 \mathrm{ng}$ of DNA template, $5 \times$ GoTaq ${ }^{\circledR}$ Flexi Buffer (Promega), $200 \mathrm{mM}$ of $\mathrm{MgCl}_{2}$, $10 \mathrm{mM}$ of dNTPs, and $25 \mu \mathrm{M}$ of each primer. The PCR reaction was performed with the MultiGene ${ }^{\mathrm{TM}}$ Gradient Thermal Cycler (Labnet) using the following set-

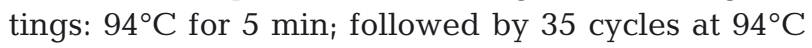
for $1 \mathrm{~min}, 57^{\circ} \mathrm{C}$ for $1 \mathrm{~min}$, and $72^{\circ} \mathrm{C}$ for $1 \mathrm{~min}$; with a final extension at $72^{\circ} \mathrm{C}$ for $10 \mathrm{~min}$.

Additional amplifications of the spacer region between the 16S and 23S rRNA genetic loci were performed with the DNA extracted from each isolate using the universal primer pair designed by Barry et al. (1991), with minor modifications. Briefly, the PCR reaction was performed using GoTaq ${ }^{\circledR}$ DNA Poly-

Table 1. Primers used to confirm Francisella noatunensis subsp. orientalis from DNA samples examined through PCRs for 16S rRNA, internal transcribed spacer (ITS), and iglC genes. F: forward; R: reverse

\begin{tabular}{|c|c|c|c|c|}
\hline Specificity & Primer/location & Sequence $\left(5^{\prime}-3^{\prime}\right)$ & mplicon size (bp) & Reference \\
\hline Eubacteria $16 \mathrm{~S}$ & $\begin{array}{l}\mathrm{UN}(27 \mathrm{~F})^{\mathrm{a}} \\
\mathrm{EB}(1487 \mathrm{R})^{\mathrm{a}}\end{array}$ & $\begin{array}{l}\text { AGA GTT TGA TCC TGG CTC AG } \\
\text { ACG GAT ACC TTG TTA CGA GTT }\end{array}$ & 1534 & Barry et al. (1990) \\
\hline Eubacteria ITS & $\begin{array}{l}\text { A1 }(1493)^{\mathrm{b}} \\
\text { B1 }(23)^{\mathrm{c}}\end{array}$ & $\begin{array}{l}\text { AGT CGT AAC AAG GTA GCC G } \\
\text { CCG TTG CCA AGG CAT CCA CC }\end{array}$ & 300 & Barry et al. (1991) \\
\hline Genus Francisella & $\begin{array}{l}\text { F1 }(149)^{\mathrm{a}} \\
\text { F5 }(1290)^{\mathrm{a}}\end{array}$ & $\begin{array}{l}\text { TAC CAG TTG GAA ACG ACT GT } \\
\text { CCT TTT TGA GTT TCG CTC C }\end{array}$ & 1100 & Forsman et al. (1994) \\
\hline $\begin{array}{l}\text { F. noatunensis subsp. } \\
\text { orientalis }\end{array}$ & $\begin{array}{l}\text { iglCF } \\
\text { iglCR }\end{array}$ & $\begin{array}{l}\text { GGG CGT ATC TAA GGA TGG TAT GAG } \\
\text { AGC ACA GCA TAC AGG CAA GCT A }\end{array}$ & 100 & Soto et al. (2010) \\
\hline
\end{tabular}


merase (Promega) in a final volume of $25 \mu \mathrm{l}$, which contained $100 \mathrm{ng}$ of total genomic DNA as a template, 5× GoTaq ${ }^{\circledR}$ Flexi Buffer (Promega), $200 \mathrm{mM}$ of $\mathrm{MgCl}_{2}, 10 \mathrm{mM}$ of dNTPs, and $25 \mu \mathrm{M}$ of the primers A1 and B1 (Table 1), which generated a product of approximately $300 \mathrm{bp}$. The cycling protocol was 1 cycle at $94^{\circ} \mathrm{C}$ for $5 \mathrm{~min}$; followed by 35 cycles at $94^{\circ} \mathrm{C}$ for $1 \mathrm{~min}, 50^{\circ} \mathrm{C}$ for $1 \mathrm{~min}$, and $72^{\circ} \mathrm{C}$ for $1 \mathrm{~min}$; with a final extension at $72^{\circ} \mathrm{C}$ for 10 min (Barry et al. 1991).

All amplified products were detected through horizontal $1.5 \%(\mathrm{w} / \mathrm{v})$ agarose gel electrophoresis for $60 \mathrm{~min}$ at $100 \mathrm{~V}$ in $1 \times$ TAE electrophoresis buffer. The products were visualized using ethidium bromide and photographed under a UV transilluminator (MiniBIS Pro Bio-Imaging Systems).

\section{Real time-PCR of the iglC gene}

To identify at the species level whether mortalities were caused by F. noatunensis subsp. orientalis, realtime PCRs were performed using the IglCR and IglCF primers described by Soto et al. (2010), together with the DNA obtained from the bacterial colonies and from splenic tissue of symptomatic fish. Real time-PCR assays were performed in a final volume of $20 \mu$ l using the SYBR ${ }^{\circledR}$ Green PCR Master Mix Kit (Applied Biosystems), which included all of the reagents needed for the PCR reactions, except for $2 \mu \mathrm{l}$ of DNA and $1 \mu \mathrm{M}$ of each primer. Samples were amplified using a One Step Applied Biosystems Thermocycler, and the PCR conditions were the same as previously described by Soto et al. (2010): $10 \mathrm{~min}$ at $95^{\circ} \mathrm{C}$; followed by 40 cycles of $15 \mathrm{~s}$ at $95^{\circ} \mathrm{C}$, and $60 \mathrm{~s}$ at $60^{\circ} \mathrm{C}$. The real-time PCR amplicon was confirmed by the melting temperature of the amplicon and by electrophoresis on $2 \%$ SeaKem ${ }^{\circledR}$ LE agarose gel and ethidium bromide staining. Melting peaks were automatically calculated using Step One software 2.0 (Applied Biosystems). Finally, the nucleotide sequences amplified using real-time PCR were confirmed through sequencing by Macrogen (Korea).

\section{Phylogenetic analysis}

The expected $1534 \mathrm{bp}$ and $300 \mathrm{bp}$ amplicons were purified using the E.Z.N.A. Gel Extraction Kit (Omega BIO-TEK) following the manufacturer's instructions, and the purified products were then sequenced by Macrogen (Korea). The resulting 16S rRNA and ITS sequences were analyzed using the Basic Local Alignment Search Tool (BLAST, http:// blast.ncbi.nlm.nih.gov/). The 16S rRNA and ITS sequences were then compared using the MEGA 5.2 program against type strain sequences of Francisella species included in the GenBank database (Tamura et al. 2011). Bootstrap values were obtained from 1000 replicates, and a maximum-likelihood consensus tree was constructed for the 16S rRNA and ITS sequences.

For comparative analyses of the sequences, only regions with the same bp lengths were considered. Analysis of the 16S sequence from Francisella strains was performed considering 14 GenBank nucleotide sequences (Table 2), and all sequences in the final dataset were adjusted to $792 \mathrm{bp}$. In the case of the ITS region, comparative analysis was performed for 11 GenBank nucleotide sequences (Table 2), which were adjusted to a $192 \mathrm{bp}$ dataset.

\section{RESULTS}

\section{Clinical signs and gross pathology of infected tilapia}

Of the analyzed fish, $20 \%$ presented pale gills, mild exophthalmia, and moderate abdominal distention, while a lesser percentage demonstrated whitish nodules in the gills, together with a shortened lamina, consolidated areas of dark coloration, necrosis, and excess mucus (Fig. 1A,B). Some individuals were emaciated and inactive, and some had an erratic swimming pattern. With the exception of scale loss, no external lesions were observed. Skin scrapes of mucus evidenced a low presence of monogenic Dactylogyrus spp. and the protozoans Trichodina spp. and Apiosoma spp. (data not shown). However, due to low quantities, these were not associated with the observed clinical conditions (i.e. francisellosis).

Internally, fish presented moderate serous ascites, adhesions in the coelomic cavity and between internal organs, and ovaries with whitish nodules. The spleen had severe splenomegaly with multiple, differently sized whitish nodules (granulomas) with diffuse distribution at different levels (Fig. 1A,C). The kidney showed larger-sized granulomas, but these did not present a defined distribution. In the liver, an irregular pale coloration was observed, with few granulomas and fibrinous adherences in the hepatic capsule (Fig. 1A). To a lesser degree, granulomas were also evidenced in the ovaries and testes (Fig. 1C,D). Imprints of the affected organs (i.e. spleen and kidney) revealed the presence of pleomorphic, Gramnegative, and intracellular coccobacillus. 
Table 2. GenBank sequences for the Francisella noatunensis subsp. orientalis strains and other bacterial species. GenBank accession numbers are listed for all 16S rDNA and internal transcribed spacer (ITS) DNA genes. Dash indicates no available information

\begin{tabular}{|c|c|c|c|}
\hline Species/strain & GenBank acc. no. & Location & Original isolation source/strain \\
\hline \multicolumn{4}{|l|}{ 16S rDNA } \\
\hline Francisella tularensis subsp. holarctica & AY968231.1 & USA & FSC 257 \\
\hline Francisella tularensis subsp. tularensis & AY968225.1 & USA & FSC 199 \\
\hline Francisella tularensis subsp. mediasiatica & AY968236.1 & USA & FSC 149 \\
\hline Cf. Francisella sp. & AF385857.2 & Taiwan & Oreochromis spp. \\
\hline Tilapia parasite & AF206675.1 & Taiwan & Tilapia spp. \\
\hline Wolbachia persica & M21292.1 & - & ATCC VR 331 \\
\hline Francisella philomiragia & Z21933.1 & USA & Homo sapiens \\
\hline Francisella noatunensis subsp. orientalis & NR_074581.1 & Indonesia & Oreochromis spp. \\
\hline Francisella noatunensis subsp. orientalis & EU683030.1 & Japan & Parapristipoma trilineatum \\
\hline Francisella noatunensis subsp. orientalis & KM393210.1 & Mexico & Oreochromis spp. \\
\hline Coxiella burnetii & AY342037.1 & Korea & Haemaphysalis longicornis \\
\hline Legionella pneumophila & M59157.1 & - & ATCC 33152 \\
\hline Piscirickettsia salmonis LF-89 & U36941.1 & Chile & Oncorhynchus kisutch \\
\hline Pseudomonas aeruginosa & M34133.1 & USA & strain 25330 \\
\hline Aeromonas salmonicida subsp. salmonicida & X60405.2 & UK & NCIMB 1102 \\
\hline Proteus vulgaris & X07652.1 & Germany & IFAM 1731 \\
\hline Agrobacterium tumefaciens & AY972446.1 & USA & Sludge \\
\hline \multicolumn{4}{|l|}{ ITS rDNA } \\
\hline Francisella tularensis subsp. holarctica & СР003862.1 & USA & FSC200 \\
\hline Francisella tularensis subsp. novicida & CP000439.1 & USA & U112 \\
\hline Francisella tularensis subsp. mediasiatica & CP000915.1 & USA & FSC147 \\
\hline Francisella philomiragia subsp. philomiragia & CP000937.1 & USA & ATCC 25017 \\
\hline Francisella noatunensis subsp. orientalis & EU683030.1 & Japan & Parapristipoma trilineatum \\
\hline Francisella noatunensis subsp. orientalis & KM393210.1 & Mexico & Oreochromis spp. \\
\hline Cf. Francisella sp. & AF385857.2 & Taiwan & Oreochromis spp. \\
\hline Francisella piscicida & DQ309246.2 & Norway & Gadus morhua \\
\hline Piscirickettsia salmonis LF-89 & AF205383.1 & Chile & Oncorhynchus kisutch \\
\hline Bacillus subtilis & JQ518353.1 & China & Chinese fermented soybean food \\
\hline Photobacterium damselae subsp. piscicida & FN646576.1 & Spain & Dicologoglossa cuneata \\
\hline
\end{tabular}

\section{Bacteriological analysis}

Bacteriological examination of spleen, liver, and kidney samples using Columbia blood agar, tryptone soya agar, brain heart infusion agar, and MacConkey agar plates did not evidence bacterial colony growths. However, pure bacterial isolates were recovered on $\mathrm{CHAB}$ plates from the majority of the sampled fish, but only 5 spleen-obtained representative isolates were studied from this outbreak.

\section{Histological analysis}

Tissue sections stained with hematoxylin and eosin presented multiple areas with severe granulomatous inflammation during distinct stages of disease progression in the liver, spleen, and kidney (Fig. 2A-C). Granulomas were also present in lesser quantities in the intestine (Fig. 2D), gills (Fig. 2E,F), heart (Fig. 2G), ovaries (Fig. 2H), and testes (data not shown). In more severe cases, wide areas of necrosis were observed, with diffuse coagulation and necrotizing vasculitis infiltrating mononuclear cells. In the majority of cases, pleomorphic bacteria were observed between necrotic material, histiocytic cells, and fibroblasts. Finally, the liver evidenced the typical lesion of poly arteritis, or granulomatous arteritis, as referenced in cases of francisellosis (Fig. 2A).

\section{Amplification and analysis of 16S rRNA and ITS sequences of Francisella spp.}

The DNA extracted from spleen and kidney samples, as well as in all DNA obtained from each isolate, produced an amplified product of approximately $1100 \mathrm{bp}$, which is consistent with the size specific to the 16S rRNA gene of Francisella genus microorganisms (Fig. 3). Sequencing analysis of the nearly complete (1100 bp) 16S rRNA gene revealed that all of the studied Mexican isolates were identical and allocated within the genus Francisella. In fact, comparative analysis of 16S rRNA from a representative Mexican isolate, coded Franc-COS1 (GenBank accession KM393210; Table 2), indicated an identity of $99.5 \%$ 

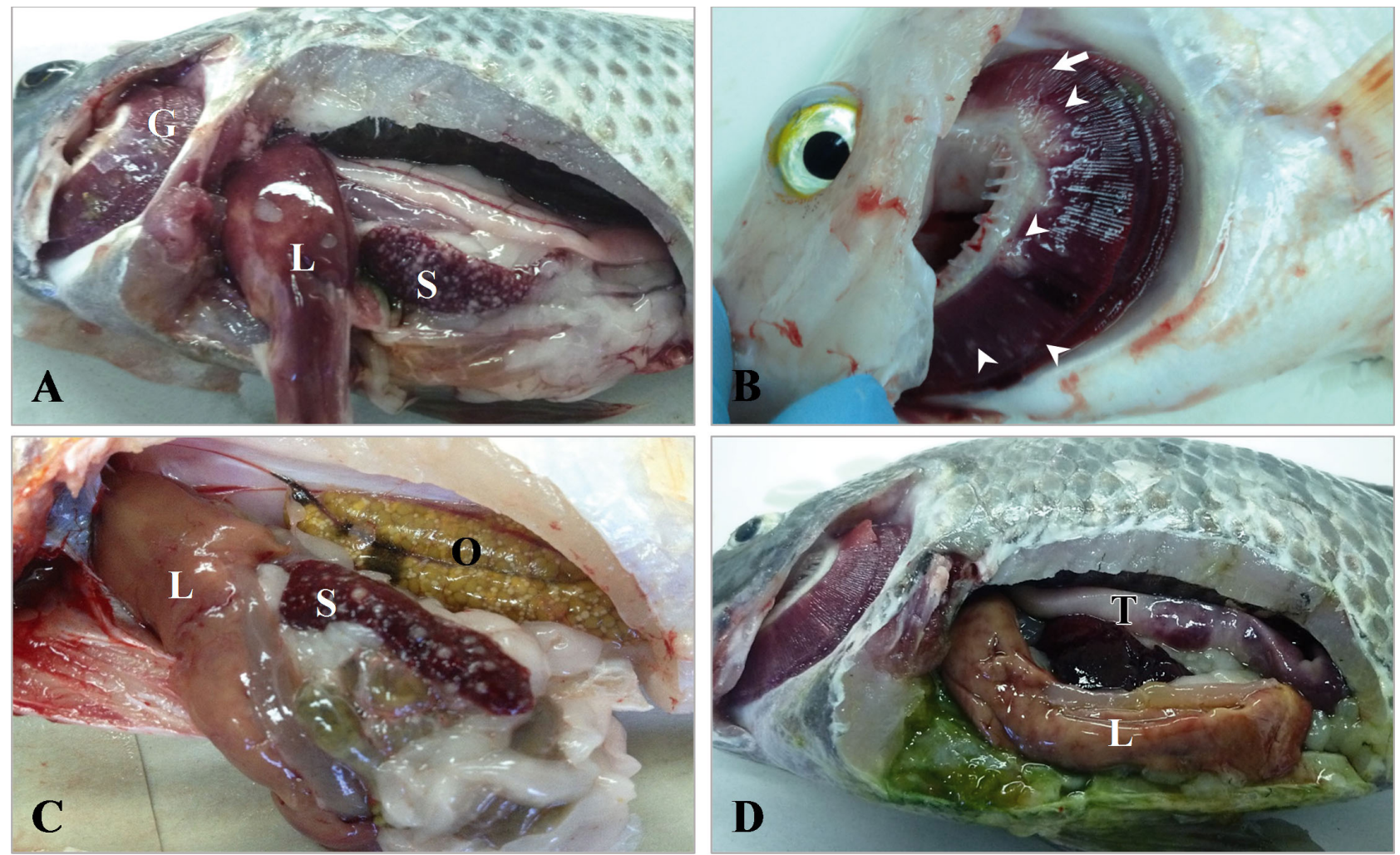

Fig. 1. Macroscopic lesions observed during necropsy of Mexican tilapia Oreochromis spp. with francisellosis. (A) Spleen (S) with severe splenomegaly and the presence of multiple, distinctly sized, whitish nodules (granulomas) with diffuse distribution. In the liver (L), an irregular pale coloration was observed with few subcapsular granulomas and fibrinous adherences in the hepatic capsule. The gills (G) were pallid and with abundant mucus. (B) Gills with whitish nodules (arrowheads) and necrotic areas (arrow). (C) Ovary (O) with the presence of multiple whitish nodules with diffuse distribution. (D) Testis (T) with whitish nodules and bleeding areas

with the following strains: F. noatunensis subsp. orientalis Ehime-1, isolated from Parapristipoma trilineatum in Japan (GenBank accession EU683030); Toba04, isolated from tilapia in Indonesia (GenBank accession NR_07581.1); and TPT-541 and CYH-2002, isolated from tilapia in Taiwan (GenBank accession AF206675 and AF385857, respectively).

The ITS primers A1 and B1 were used to confirm that all isolates were correctly assigned to the genus Francisella. The Mexican Franc-COS1 isolate presented $98.78 \%$ identity with the ITS sequence of the Ehime-1 F. noatunensis subsp. orientalis strain. Additionally, $97 \%$ identity was detected with F. philomiragia subsp. philomiragia (GenBank accession CP000937.1).

\section{Phylogenetic tree}

In the case of the16S rRNA gene, all sequences of the Francisella genus were grouped in the same clade, and the Franc-COS1 isolate was the most closely related to a subclade within $F$. noatunensis subsp. orientalis NR_074581.1, presenting a bootstrap support of $99.5 \%$ (Fig. 4). Phylogenetic analysis of the ITS region placed the Mexican isolate within the Francisella clade, where a close relationship was found with F. noatunensis subsp. orientalis EU683030.1, presenting a bootstrap support of $85 \%$ (Fig. 5). From this information, a sequence identity matrix was constructed to estimate evolutionary divergences. Comparison of the 16S rRNA and ITS sequences of Francisella spp. did not show any differences between the analyzed regions of the $F$. noatunensis subsp. orientalis strain, instead presenting $100 \%$ identity.

\section{Real time-PCR amplification of the iglC gene}

Specific real time-PCR amplification of iglC confirmed the presence of $F$. noatunensis subsp. orientalis, with threshold cycle values (Ct) of 16 to 20 for 

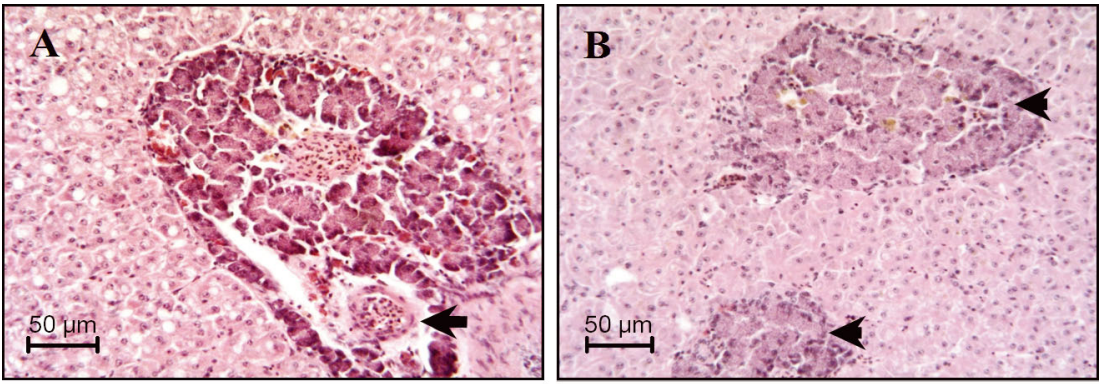

Fig. 2. Histological analysis of organs in Mexican tilapia Oreochromis spp. affected by francisellosis. (A) Liver with multifocal granulomatous hepatitis. Necrotic area surrounded by blood vessels, with infiltration by mononuclear inflammatory cells on the periphery and wall of 1 vessel (arteritis, arrow). (B) Multifocal necrosis in the liver parenchyma, with infiltration of mononuclear cells on the periphery (arrows). (C) Spleen with multifocal granulomatous splenitis (arrows). (D) Intestine with multifocal granulomatous enteritis, with granulomas in the apex of intestinal villi (arrows). (E) Histological section of a gill showing focal granulomatous branchitis at the base of the secondary gill lamellae, characterized by clusters of macrophages. (F) Magnification of the square in (E). (G) Heart with focal granulomatous myocarditis composed of clusters of macrophages among cardiac fibers. (H) Ovary showing a granulomatous perivasculitis oophoritis in the cortex, characterized by the presence of perivascular clusters of macrophages.

Staining performed with $\mathrm{H} \& \mathrm{E}$

Fig. 3. PCR detection of Francisella species from DNA samples obtained from pure bacterial cultures and symptomatic tilapia (Oreochromis spp.) spleen tissue. Each line shows the amplification using the primers described by Forsman et al. (1994), with a size of 1100 bp. MW: molecular ruler; -: negative control (no DNA); +: positive control (DNA extracted from $F$. noatunensis subsp. orientalis)

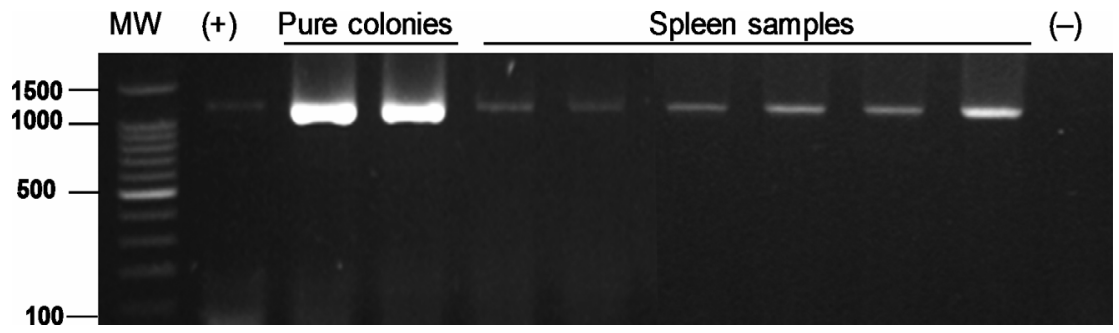

DNA from pure colonies. Positive reactions showed a single melting peak, with a melting temperature of $75.4^{\circ} \mathrm{C}$. The corresponding $88 \mathrm{bp}$ amplicon (Fig. 6A) was confirmed by nucleotide sequencing from both bacterial cultures and infected tissues, showing $100 \%$ identity with iglC from the $F$. noatunensis subsp. orientalis reference strain LADL 07-285A (Soto et al. 2010) (Fig. 6B). 


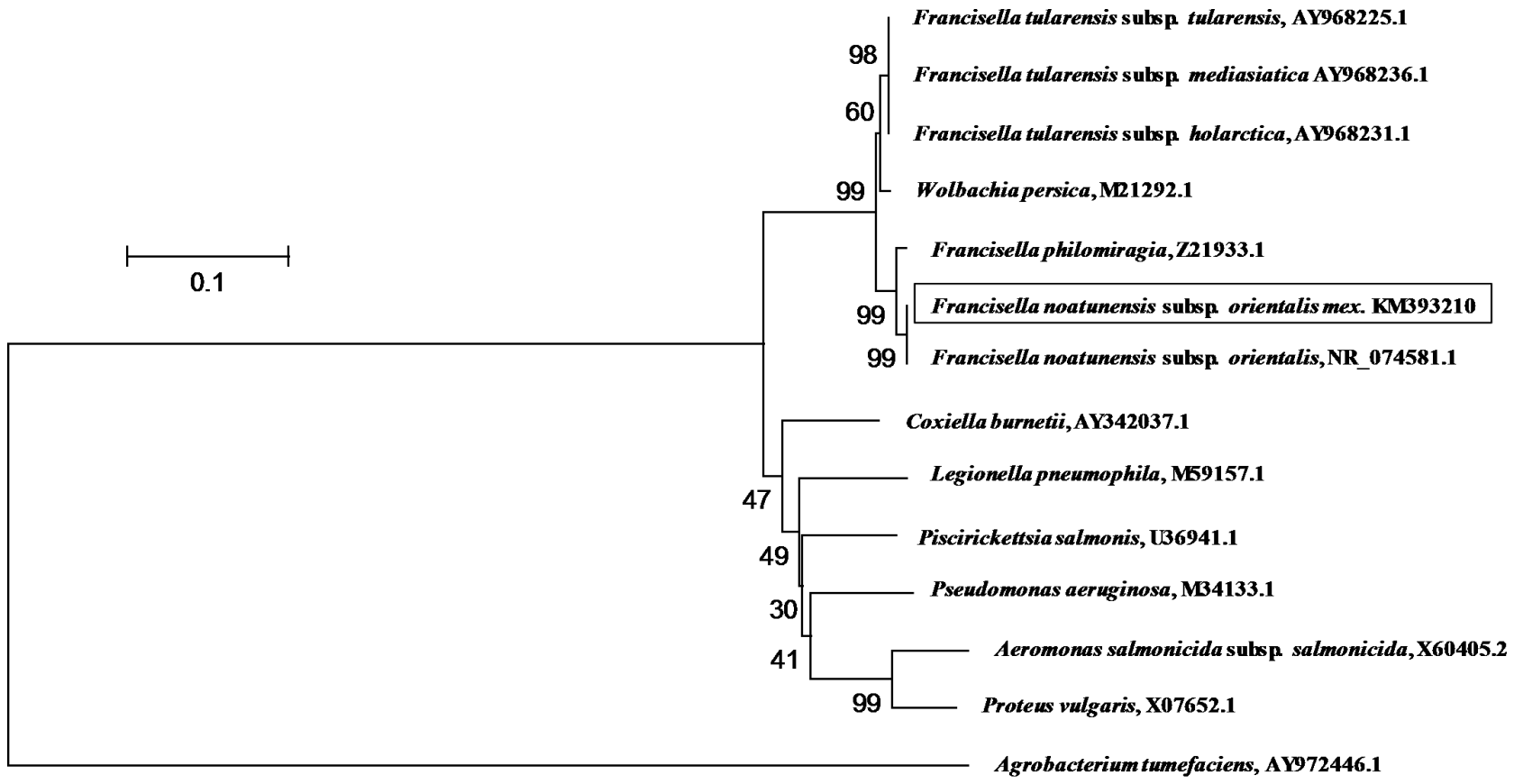

Fig. 4. Neighbor-joining tree based on 16S RNA sequences (792 bp), showing the phylogenetic position of a representative Mexican Francisella noatunensis subsp. orientalis isolate coded as Franc-COS1 (accession number KM393210) obtained from diseased tilapia Oreochromis spp. (inside the rectangle). Bootstrap values (>50\%) based on 1000 replications are shown at the 3 nodes of the tree. Agrobacterium tumefaciens was used as an outgroup. Bar $=10$ substitutions per 100 nucleotides

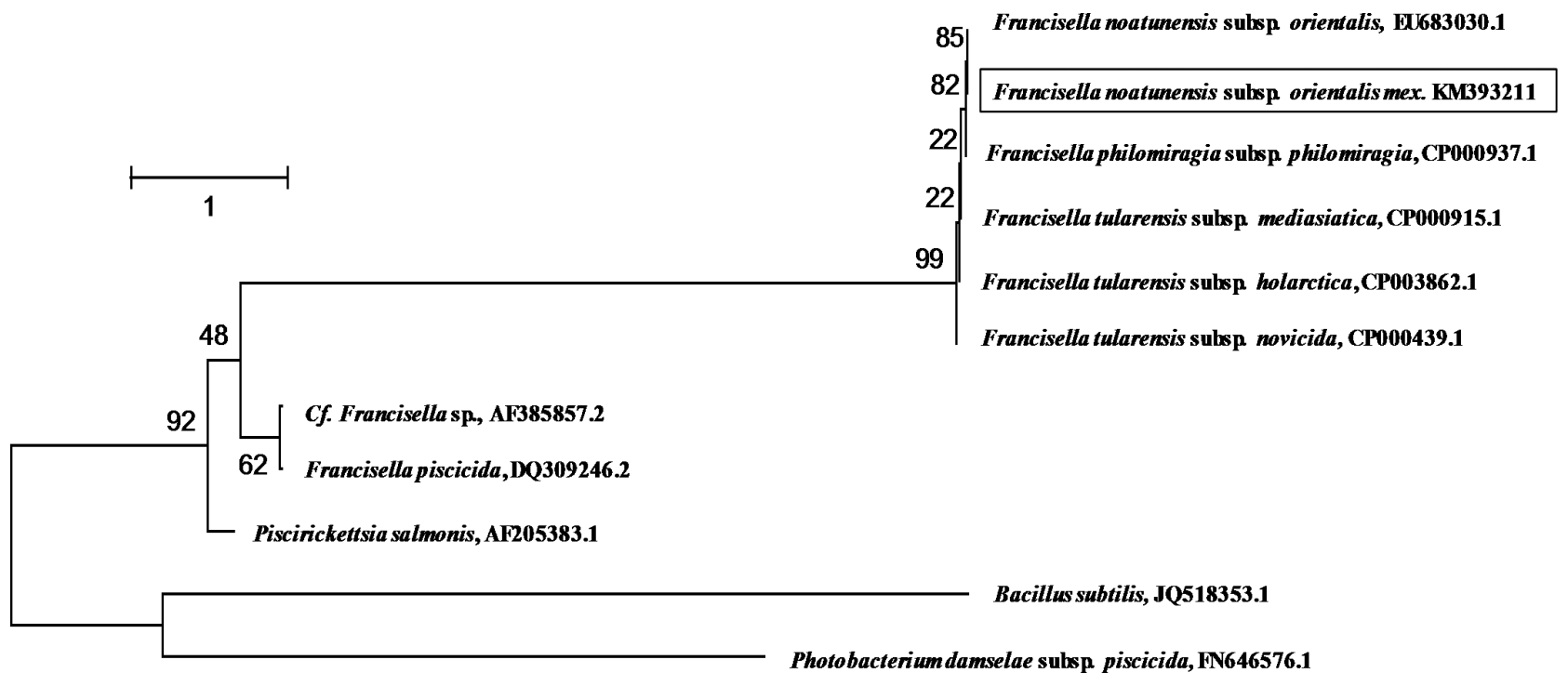

Fig. 5. Neighbor-joining tree based on internal transcribed spacer (ITS) gene sequences (194 bp), showing the relationship between the Mexican Francisella noatunensis subsp. orientalis isolate Franc-COS1 and other species of the genus Francisella. Bootstrap values from 1000 replicates appear at the nodes at an identity $>50 \%$. The other microorganisms were used as an outgroup

\section{DISCUSSION}

Granulomatous lesions in fish can be caused by a number of pathogens (Colquhoun \& Duodu 2011). In the present study, macroscopic, microscopic, and mo- lecular analyses confirmed that the analyzed fish were infected with Francisella spp., a known genus of intracellular microorganisms capable of causing disease in tilapia (Mauel et al. 2003, 2005, Birkbeck et al. 2007, Soto et al. 2009b, Colquhoun \& Duodu 2011). 


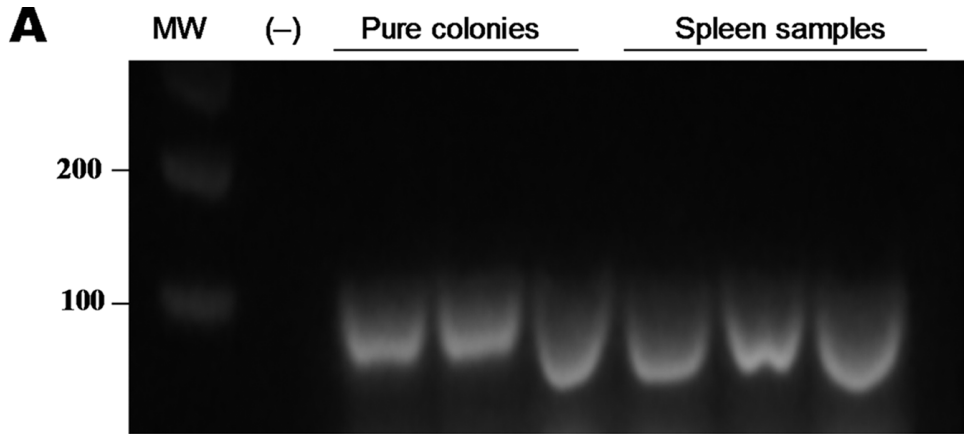

\section{B LADL07-285A igic ATATCTATTg ATGGgCTCAC AACTTCACAA GgTAGCTTGC CTGTATGCTG TGCT Franc-COS1 iglC ATATCTATTG ATGGGCTCAC AACTTCACAA GGTAGCTTGC CTGTATGCTG TGCT}

Fig. 6. (A) Specific PCR product amplified with iglCF and iglCR primer sets for Francisella noatunensis subsp. orientalis using DNA samples obtained from pure bacterial colonies and infected tilapia (Oreochromis spp.) spleen tissue. Lane MW: 100 bp DNA molecular marker; Lane (-); negative control (no DNA). (B) Sequence comparisons of specific PCR products from colonies of the Mexican isolate Franc-COS1 and of the iglC gene from strain LADL 07-285A (GenBank FJ386388) via sequence alignments using ClustalW

In addition to the presence of multifocal whitish nodules in the internal organs of diseased fish, francisellosis is characterized by the absence or limited presentation of clinical signs and external lesions. Indeed, although the analyzed fish presented cachexia, anorexia, anemia, and slow swimming, these fish did not show the hemorrhaging, external ulcerations, or muscular damage described by Mauel et al. (2007); the abdominal distension observed in prior studies (Soto et al. 2011) was also not marked in our current observations. However, it is worth noting that the presence of clinical signs and lesions, as well as mortality rate, can vary depending on factors involved in the triad of epizootiology and on the stage in which the disease is analyzed.

According to the case history, when the tilapia farm requested disease diagnosis, infection was already chronic and had affected cultures for nearly 6 mo. Therefore, at the time of analyses, mortality was not highly manifested. Nevertheless, mortality reached a rate of $40 \%$ during the initial outbreak, which is in agreement with previous reports in other species (Kamaishi et al. 2005, Olsen et al. 2006, Ostland et al. 2006).

Water temperature is a determining factor in the clinical manifestation and mortality rate of aquatic diseases (Mauel et al. 2007), with higher mortalities occurring at temperatures between 21.5 and $26.3^{\circ} \mathrm{C}$ (Mauel et al. 2005). Considering this, the mortality presented by the currently described outbreak could be explained by the $26^{\circ} \mathrm{C}$ water temperature at the time of the outbreak. Another critical factor in the transmission of disease is water recirculation in high biomass systems, which favor contact between fish and increase waste concentration (Jeffery et al. 2010). These situations were all present at the affected farm, where high mortality was also associated with the constant handling of reproductive animals (Mauel et al. 2003, 2007, Soto et al. 2009a). While vertical transmission of this disease has not been demonstrated, offspring have been diagnosed, with infection possibly resulting through water recirculation and high biomass (Colquhoun \& Duodu 2011). The origin of francisellosis in Mexico could not be determined. It is important to note that, in the months prior to the outbreak, a tilapia lot originating from Central America was introduced into the subsequently affected Mexican farm. The number of farms and the areas where that fish group was introduced are unknown.

Francisellosis is associated with the formation of whitish nodules, predominantly in the spleen and kidney, although these can be present in other organs (Mauel et al. 2007, Colquhoun \& Duodu 2011, Soto et al. 2011). In the current study, these nodules were present in all examined organs, with the exception of the brain. Histological analyses of lesions in the affected tissues were consistent with the reports by Mauel et al. (2003, 2007), who characterized granulomas as an infiltrate mix containing vacuolated macrophages, lymphocytes, and neutrophils in a necrotic area where Gram-negative pleomorphic bacteria are present and are surrounded by a fibrous capsule. In the analyzed tissue samples, necrotizing vasculitis and thrombosis of the liver were among the notable findings, which is in addition to recording typical granulomatous arteritis with infiltration of macrophages, lymphocytes, and necrotic cells. All of 
these lesions have been previously described (Mauel et al. 2007, Colquhoun \& Duodu 2011, Soto et al. 2011).

The causative agent of the lesions in tilapia was confirmed in 10 fish sampled using specific PCR amplification of the 16S rRNA from the genus Francisella. Forsman et al. (1994) indicated that 31 Francisella strains have been identified. Phylogenetic analyses using the nearly complete 16S rRNA gene sequence from the Mexican tilapia isolate FrancCOS1 resulted in greater than $99.5 \%$ identity with 2 F. noatunensis subsp. orientalis strains (Ethime-1 and Toba04). These strains were obtained from different host species, including tilapia. Furthermore, ITS analyses confirmed the phylogenetic relationship of the Franc-COS1 isolate with the Japanese F. noatunensis subsp. orientalis Ehime-1 strain.

It is important to note that since 2006, the identification of novel Francisella species recovered from distinct fish species has not been easy; therefore, these isolates are classified as Francisella-like (Hsieh et al. 2006, Soto et al. 2010). Some of these isolates have been classified as belonging to F. noatunensis within the new subspecies orientalis (Mikalsen et al. 2007, Ottem et al. 2007, 2009, Soto et al. 2011).

The single copy of the IglC gene in F. noatunensis subsp. orientalis has allowed for the development of a highly specific diagnostic test (Soto et al. 2010) used to confirm the identity of representative Mexican Franc-COS1 isolates as F. noatunensis subsp. orientalis. This finding is very important considering that distinct species within the genus Francisella share a high percentage of identity (>95\%) at the level of the 16S rRNA gene sequence. In fact, comparative analysis of the $16 \mathrm{~S}$ gene sequence from the $F$. noatunensis subsp. orientalis LADL 07-285A and F. noatunensis subsp. noatunensis showed more than $99 \%$ identity between strains, but when comparing the results for $i g l C$, this identity is reduced to $90 \%$. Additionally, sequence alignment of the PCR product specific to iglC in the Mexican isolate against sequences contained in GenBank demonstrated 100\% identity with the same target described in F. noatunensis subsp. orientalis (Soto et al. 2010), thus confirming for the first time the presence of this pathogenic agent in tilapia farmed in Mexico. Additional studies are needed to evaluate the risk for Mexican aquaculture. A fish challenge experiment should be performed to fulfill Koch's postulates and to establish prevention and control strategies against the development of infection in the promising Mexican tilapia industry.
Acknowledgements. This work was performed with the support and resources provided by the grants Fondo semilla UAEM-UAch Clave 3334/2012FSChF and FONDAP/CONICYT 15110027. We also thank Dr. John Hawke of Louisiana State University for his contributions.

\section{LITERATURE CITED}

Barry T, Powell R, Gannon F (1990) A general method to generate DNA probes for microorganisms. Biotechnology 8:233-236

Barry T, Colleran G, Glennon M, Dunican LK, Gannon F (1991) The 16S/23S ribosomal spacer region as a target for DNA probes to identify eubacteria. PCR Methods Appl 1:51-56

Birkbeck TH, Bordevik M, Froystad MK, Baklien A (2007) Identification of Francisella sp. from Atlantic salmon, Salmo salar L., in Chile. J Fish Dis 30:505-507

Colquhoun DJ, Duodu S (2011) Francisella infections in farmed and wild aquatic organisms. Vet Res 42:47

Conapesca (Comisión Nacional de Acuicultura y Pesca) (2012) Anuario estadístico de acuicultura y pesca 2012. Comisión Nacional de Acuicultura y Pesca, Secretaría de Agricultura Ganadería Desarrollo Rural Pesca y Alimentación. Available at www.siap.gob.mx/wp-content/uploads/2013/ 12/Anuario_2012.pdf (accessed 28 May 2013)

Fernando CH, Furtado JI, Gussev AV, Hanek G, Kakong SA (1972) Methods for the study of freshwater fish parasites, $1^{\text {st }}$ edn. Biology series. University of Waterloo, Waterloo, ON

Fitzsimmons K (2000) Tilapia aquaculture in Mexico. In: Costa-Pierce BA, Rakocy JE (eds) Tilapia aquaculture in the Americas, Vol 2. The World Aquaculture Society, Baton Rouge, LA, p 171-183

Forsman M, Sandstrom G, Sjostedt A (1994) Analysis of 16S ribosomal DNA sequences of Francisella strains and utilization for determination of the phylogeny of the genus and for identification of strains by PCR. Int J Syst Bacteriol 44:38-46

Hsieh CY, Tung MC, Tu C, Chang CD, Tsai SS (2006) Enzootics of visceral granulomas associated with Francisella-like organism infection in tilapia (Oreochromis spp.). Aquaculture 254:129-138

Jeffery KR, Stone D, Feist SW, Verner-Jeffreys DW (2010) An outbreak of disease caused by Francisella sp. in Nile tilapia Oreochromis niloticus at a recirculation fish farm in the UK. Dis Aquat Org 91:161-165

Kamaishi T, Fukuda Y, Nishiyama M, Kawakami H, Matsuyama T, Yoshinaga T, Oseko N (2005) Identification and pathogenicity of intracellular Francisella bacterium in three-line grunt Parapristipoma trilineatum. Fish Pathol 40:67-71

Mauel MJ, Miller DL, Frazier K, Liggett AD, Styer L, Montgomery-Brock D, Brock J (2003) Characterization of a piscirickettsiosis-like disease in Hawaiian tilapia. Dis Aquat Org 53:249-255

Mauel MJ, Miller DL, Styer E, Pouder DB, Yanong RP, Goodwin AE, Schwedler TE (2005) Occurrence of piscirickettsiosis-like syndrome in tilapia in the continental United States. J Vet Diagn Invest 17:601-605

Mauel MJ, Soto E, Moralis JA, Hawke J (2007) A piscirickettsiosis-like syndrome in cultured Nile tilapia in Latin America with Francisella spp. as the pathogenic agent. J Aquat Anim Health 19:27-34 
Mikalsen J, Olsen AB, Tengs T, Colquhoun DJ (2007) Francisella philomiragia subsp. noatunensis subsp. nov., isolated from farmed Atlantic cod (Gadus morhua L.). Int J Syst Evol Microbiol 57:1960-1965

Nylund A, Ottem KF, Watanabe K, Karlsbakk E, Krossoy B (2006) Francisella sp. (Family Francisellaceae) causing mortality in Norwegian cod (Gadus morhua) farming. Arch Microbiol 185:383-392

Olsen AB, Mikalsen J, Rode M, Alfjorden A and others (2006) A novel systemic granulomatous inflammatory disease in farmed Atlantic cod, Gadus morhua L., associated with a bacterium belonging to the genus Francisella. J Fish Dis 29:307-311

Ortega C, Valladares B (2015) Analysis on the development and current situation of rainbow trout (Oncorhynchus mykiss) farming in Mexico. Rev Aquacult 0:1-9

Ostland VE, Stannard JA, Creek JJ, Hedrick RP, Ferguson HW, Carlberg JM, Westerman ME (2006) Aquatic Francisella-like bacterium associated with mortality of intensively cultured hybrid striped bass Morone chrysops $\times$ M. saxatilis. Dis Aquat Org 72:135-146

Ottem KF, Nylund A, Karlsbakk E, Friis-Moller A, Krossoy B, Knappskog D (2007) New species in the genus Francisella (Gammaproteobacteria; Francisellaceae); Francisella piscicida sp. nov. isolated from cod (Gadus morhua). Arch Microbiol 188:547-550

Editorial responsibility: Sven Klimpel,

Frankfurt, Germany
Ottem KF, Nylund A, Karlsbakk E, Friis-Moller A, Kamaishi T (2009) Elevation of Francisella philomiragia subsp. noatunensis Mikalsen et al. (2007) to Francisella noatunensis comb. nov. [syn. Francisella piscicida Ottem et al. (2008) syn. nov.] and characterization of Francisella noatunensis subsp. orientalis subsp. nov., two important fish pathogens. J Appl Microbiol 106:1231-1243

Soto E, Fernandez D, Hawke JP (2009a) Attenuation of the fish pathogen Francisella sp. by mutation of the $\mathrm{iglC}^{*}$ gene. J Aquat Anim Health 21:140-149

Soto E, Hawke JP, Fernandez D, Morales JA (2009b) Francisella sp., an emerging pathogen of tilapia, Oreochromis niloticus (L.), in Costa Rica. J Fish Dis 32:713-722

Soto E, Bowles K, Fernandez D, Hawke JP (2010) Development of a real-time PCR assay for identification and quantification of the fish pathogen Francisella noatunensis subsp. orientalis. Dis Aquat Org 89:199-207

Soto E, Baumgartner W, Wiles J, Hawke JP (2011) Francisella asiatica as the causative agent of piscine francisellosis in cultured tilapia (Oreochromis sp.) in the United States. J Vet Diagn Invest 23:821-825

> Tamura K, Peterson D, Peterson N, Stecher G, Nei M, Kumar S (2011) MEGA5: molecular evolutionary genetics analysis using maximum likelihood, evolutionary distance, and maximum parsimony methods. Mol Biol Evol 28: 2731-2739

Submitted: August 6, 2015; Accepted: April 4, 2016

Proofs received from author(s): July 5, 2016 